\title{
Early diagnosis and management of maternal ureterohydronephrosis during pregnancy
}

\author{
ELENA CICIU ${ }^{1}$, ANA-MARIA PAȘATU-CORNEA ${ }^{1 *}$, LUCIAN CRISTIAN PETCU $^{2 *}$ and LILIANA-ANA TUŢA $\breve{1}^{1,3,4}$ \\ ${ }^{1}$ Department of Nephrology, Constanta County Emergency Hospital, 900591 Constanţa; \\ ${ }^{2}$ Department of Biophysics and Biostatics, Faculty of Dental Medicine; ${ }^{3}$ Department of Clinical Medical Sciences, \\ Faculty of General Medicine; ${ }^{4}$ Doctoral School, Faculty of Medicine, \\ 'Ovidius' University of Constanţa, 900527 Constanţa, Romania
}

Received August 13, 2021; Accepted September 13, 2021

DOI: $10.3892 /$ etm.2021.10949

\begin{abstract}
Maternal ureterohydronephrosis (UHN) is a common anatomical change during the evolution of pregnancy, diagnosed especially after the 20th week of pregnancy. The aim of the present study was to evaluate the stages of UHN during pregnancy, depending on the gestational age, and to monitor the symptomatology and the adequate management. A total of 58 pregnant women with UHN, hospitalized in the Constanta County Emergency Hospital, were included in the present study, and had nephrological monitoring using ultrasound examination. Right UHN was observed in all cases and left UHN was observed in only $67.24 \%$ of the cases. Regarding the gestational age, right UHN grade III was most commonly seen between 27 and 31 weeks of pregnancy (48.6\% of total right UHN grade III from the studied group). The data showed that gestational age and grade of UHN had a highly dependent association in the studied group. The majority of our patients $(67.24 \%)$ were symptomatic, and the most common complaint on presentation was lumbar pain. According to the visual analog scale (VAS) of the lumbar pain, the group could be distributed as follows: $17.24 \%$ with severe pain, $36.21 \%$ with moderate pain and $13.79 \%$ with mild pain. Eight pregnant women (13.79\%) from the present study developed UHN due to passage of a ureteral stone, although the majority of the patients experienced complications with urinary tract infection and acute kidney injury. In addition, 97\% of the symptomatic UHN responded to conservatory treatment and only 2 patients $(3.45 \%)$ with severe symptomatic UHN needed ureteral stent insertion. Data analysis was performed using
\end{abstract}

Correspondence to: Dr Ana-Maria Pașatu-Cornea, Department of Nephrology, Constanta County Emergency Hospital, 145 Tomis Boulevard, 900591 Constanţa, Romania

E-mail: anamarycornea@gmail.com

*Contributed equally

Key words: pregnancy, ureterohydronephrosis, gestational age, lumbar pain, ureteral stent
IBM SPSS Statistics 23. The study highlighted the existence of an association between gestational age and UHN grading.

\section{Introduction}

During pregnancy, the pyelocaliceal system undergoes volume changes by pyelocaliceal dilation of approximately $15 \mathrm{~mm}$ for the right kidney and $5 \mathrm{~mm}$ for the left kidney (1). Maternal ureterohydronephrosis is one of the most common anatomical changes during pregnancy. It occurs in 43-100\% of pregnant women, being more evident in the third trimester (2). Anatomical changes of the pyelocaliceal system begin in the second month of pregnancy and can reach a $2-\mathrm{cm}$ dilation. Over $85 \%$ of pregnant women have the right ureter constantly more dilated than the left ureter (3). Some researchers suggest that the dilation on the right side is caused by mechanical compression due to an enlarged uterus and the position of the right ureter in relation with iliac and ovarian vessels. The left ureter has a greater angle in the pelvic region, which is parallel with pelvic vessels. This asymmetry in the right and left ureteral dilation could not be attributed to hormonal changes (3).

The aim of the present study was to evaluate the stages of UHN during pregnancy, depending on the gestational age, and to monitor the symptomatology and adequate management. The study highlighted the existence of an association between gestational age and UHN grading.

\section{Patients and methods}

Patient details. The aim of the present study was to investigate the relationship between the gestational age, the stage of UHN and symptomatology, and to evaluate the proper management of this special condition, mainly in the presence of complications. The values for gestational age were between 23 and 38 weeks of pregnancy, with a mean age of pregnancy of 30.98 weeks and standard deviation of 4.14 weeks.

The study included 58 pregnant women (age range, 16-45 years of age with a mean age of 30.25 years) with UHN followed for 1 year (January 2017-January 2018), following hospitalization at the Departments of Nephrology and Obstetrics and Gynecology of Constanta County Emergency Hospital. Biological (from blood and urine samples) and 
imagistic data were collected using mainly abdominal ultrasonography. Magnetic resonance imaging (MRI) was performed only in a few, exceptional cases. Some pregnant women needed reassessments during the same hospitalization due to association of complications.

The present study was approved by the local Ethics Commission of Constanta County Emergency Hospital, Romania (no. 3/24.02.2017).

Methods. Information was carefully collected and recorded in the database, including clinical-biological and imaging data, diagnosis, therapy approached, gestational age, age of pregnant women, past medical disease and complications. As an imaging method, ultrasound (Hitachi-ProSound F37 ultrasound device), which is a rapid, reliable, accessible diagnostic tool and does not involve the risk of irradiation for the mother and fetus was utilized. Information was collected on the size and position of the kidneys, the degree of hydronephrosis and the echogenicity of the renal parenchyma. The degree of hydronephrosis was classified as follows: i) grade I: minimal changes in urinary stasis; ii) grade II: slight dilation of the renal pelvis involving major calyces; iii) grade III: moderate dilation of the renal pelvis involving major and minor calyces; iv) stage IV: severe dilation with compression on the renal parenchyma (4).

Acute kidney injury (AKI) was defined as oliguria and an increase in serum creatinine of $>0.3 \mathrm{mg} / \mathrm{dl}$ in over $48 \mathrm{~h}$ (Kidney Disease Improving Global Outcomes definition).

Statistical analysis. The results of the present study were analyzed with IBM SPSS Statistics 23 program (IBM Corp.). The procedures used were descriptive statistics (for characterizing discrete and continuous variables defined in the database), graphs, non-parametric statistical tests (Chi-squared test for the association, the $\mathrm{z}$ test to compare two proportions). The significance level used was $\alpha=0.05$. A P-value less than $\alpha$ and a test statistic that falls in the critical region were the reason for rejecting the null hypothesis in favor of the alternative hypothesis.

\section{Results}

UHN and gestational age. A total of 58 pregnant women presenting with different degrees of symptomatic UHN were included in the studied group. Of these, 14 cases $(24.14 \%)$ were in the second trimester of pregnancy and $44(75.86 \%)$ in the third trimester of pregnancy. According to the location and grade of UHN, 39 women (67.24\%) out of 58 had left UHN (17 cases with grade I and 22 cases with grade II) and all 58 women had right UHN (21 cases with grade II and 37 cases grade III). Distribution of the cases with UHN (left/right) depending on grade (I, II, III) is shown in Figs. 1 and 2.

The values for gestational age were between 23 and 38 weeks of pregnancy, with a mean age of pregnancy of 30.98 weeks and standard deviation of 4.14 weeks. According to gestational age, 10 pregnant women were in the 22- to 26-week group, 23 women in the 27- to 31-week group, 18 women in the 32- to 36-week group and 7 women in the 37- to 41-week group. Distribution of the 58 cases according to gestational age (weeks) is shown in Fig. 3.
Distribution of pregnant women in the study group according to the right UHN grade vs. gestational age is presented in Fig. 4. A significant association $\left(\chi_{\text {calc }}^{2}=23.741\right.$, $\mathrm{df}=3, \mathrm{P}<0.001<\alpha=0.05$, Chi-squared test for association) was found between the gestational age and the right UHN grade. From the 58 pregnant women with right UHN, 21 (36.2\%) of them were of right UHN grade (II) and distributed according to the gestational age as follows: $17.2 \%$ (10 pregnant women) in the 22- to 26-week group, $8.6 \%$ (5 pregnant women) in the 27 - to 31 -week group, and $10.3 \%$ (6 pregnant women) in the 32- to 36-week group. The highest proportion of pregnant women with right UHN grade (II) was associated with the 22to 26-week group $(\mathrm{P}<0.05)$ which makes this gestational age interval more specific for right UHN grade (II). The remaining 37 (63.8\%) pregnant women had right UHN grade (III): $31.0 \%$ (18 pregnant women) in the 27- to 31-week group, $20.7 \%$ (12 pregnant women) in the 32- to 36-week group and $12.1 \%$ (7 pregnant women) in the 37- to 41-week group. As we previously stated, the highest proportion of pregnant women with right UHN grade (III) was associated with the 27- to 31-week group $(\mathrm{P}<0.05)$ which makes this gestational age interval more specific for right UHN grade (III). These data suggest that right UHN grade increases with gestational age.

Distribution of pregnant women in the study group according to the left UHN grade vs. gestational age is presented in Fig. 5. A significant association $\left(\chi_{\text {calc }}^{2}=12.006\right.$, $\mathrm{df}=2, \mathrm{P}=0.002<\alpha=0.05, \mathrm{CHI}$-squared test for association) was found between the gestational age (weeks) and the left UHN grade. From the 39 pregnant women with left UHN, 17 (43.6\%) of them had left UHN grade (I) and were distributed according to the gestational age as follows: $35.9 \%$ (14 pregnant women) in the 27- to 31-week group and 7.7\% (3 pregnant women) in the 32- to 36-week group. The highest proportion of pregnant women with left UHN grade (I) was associated with the 27- to 31 -week group $(\mathrm{P}<0.05)$ which makes this gestational age interval more specific for left UHN grade (I). The remaining $22(56.4 \%)$ pregnant women had left UHN grade (II): $15.4 \%$ (6 pregnant women) in the 27- to 31 -week group, $33.3 \%$ (13 pregnant women) in the 32to 36 -week group and $7.7 \%$ ( 3 pregnant women) in the 27 - to 31-week group. As previously stated, the highest proportion of pregnant women with right UHN grade (II) was associated with the 32- to 36 -week group $(\mathrm{P}<0.05)$ which makes this gestational age interval more specific for left UHN grade (II). These data suggest that left UHN grade increases with gestational age.

Parity. The distribution of the studied group depending on parity is shown in Fig. 6. A total of 37 women (63.79\%) were primiparous and $21(36.21 \%)$ were multiparous.

Intensity of lumbar pain. From the 58 pregnant women, $67.24 \%$ (39 cases) had lumbar pain as clinical manifestation and motif of presentation to the hospital. According to the intensity of lumbar pain: 19\% of women did not complain about lumbar pain, 8 (13.79\%) women had mild lumbar pain, $21(36.21 \%)$ women had moderate lumbar pain and 10 (17.24\%) women had severe lumbar pain. Distribution of the studied group depending on the intensity of lumbar pain (VAS scale) is shown in Fig. 7. 


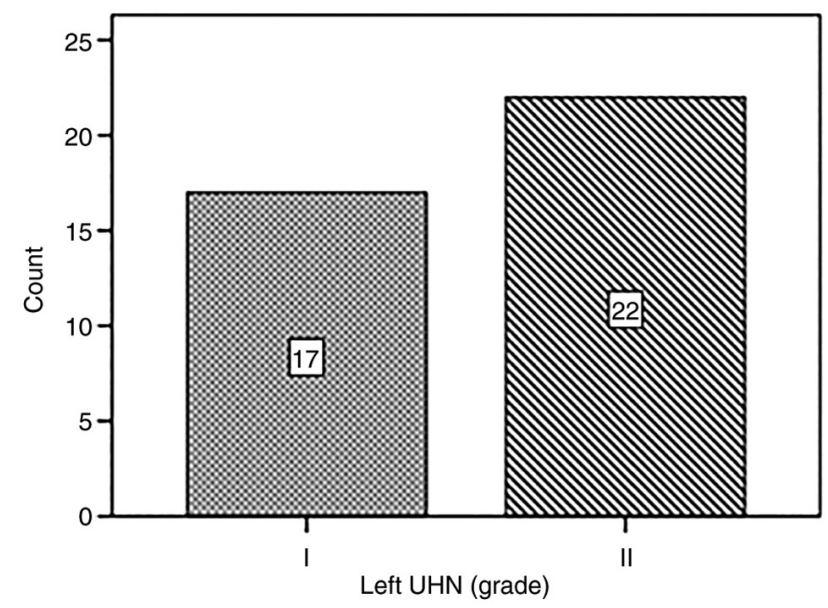

Figure 1. Distribution of cases with left UHN according to grade (I, II, III). UHN, ureterohydronephrosis.

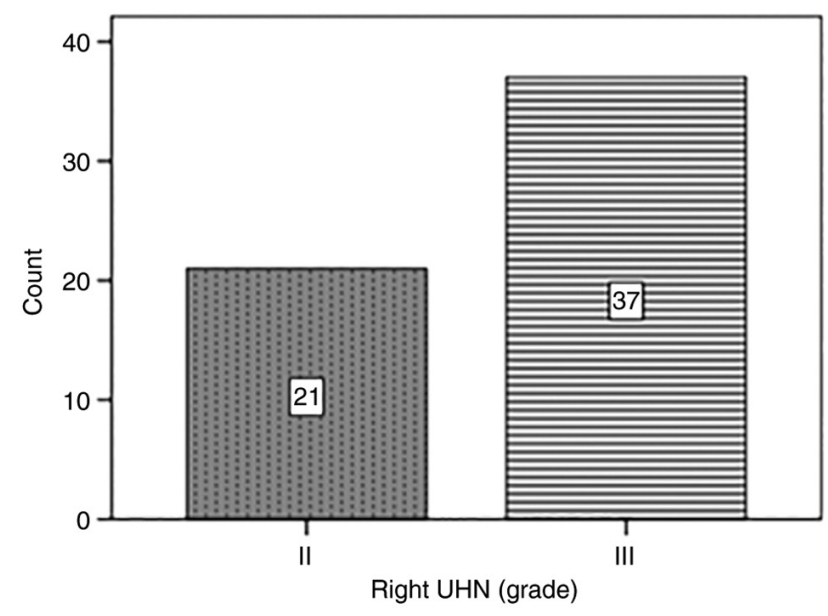

Figure 2. Distribution of cases with right UHN according to grade (I, II, III). UHN, ureterohydronephrosis.

Clinical evolution of UHN. In the studied group, UHN was associated with complications, including upper urinary tract infection (UTIs) (79.31\%; 46 cases) or acute kidney injury (12.07\%; 7 cases). According to clinical manifestations of UTIs, from the 46 pregnant women, $8(13.79 \%)$ cases had asymptomatic bacteriuria (AB), $5(8.62 \%)$ cases had acute cystitis (AC), 18 (31.03\%) cases had recurrent urinary tract infections (Rec. UTIs) and 15 (25.86\%) cases had acute pyelonephritis (AP). Depending on clinical manifestations of the urinary tract infection, pregnant women could be distributed as shown in Fig. 8.

Therapeutic management. Two patients with AKI recovered after ureteral stent insertion, 2 after withdrawal of NSAIDs and small doses of corticosteroids and 3 needed emergency delivery. Conservatory treatment was administered to all the patients and was represented by analgesics, antibiotics, adjuvant therapy and hydration. Additionally, $96.55 \%$ (56 cases) of the studied group, responded to the conservatory therapy and the symptomatology was ameliorated in those 56 cases, only 2 cases needed ureteral stent insertion.

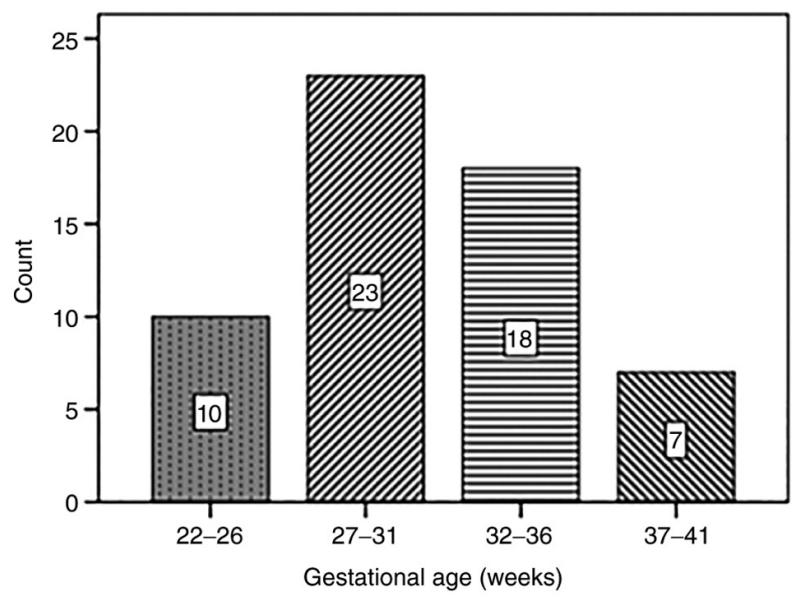

Figure 3. Distribution of cases according to gestational age (weeks).

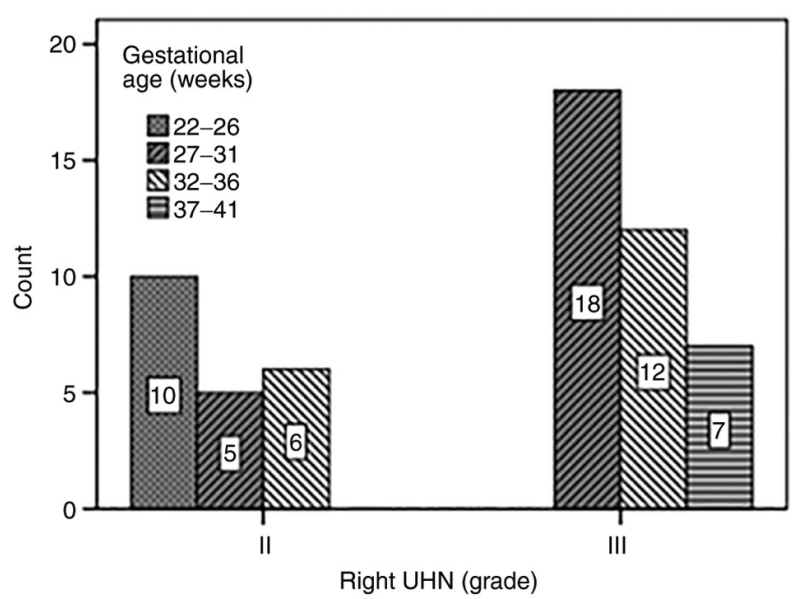

Figure 4. Distribution of right UHN depending on grade and gestational age (weeks). UHN, ureterohydronephrosis.

\section{Discussion}

Hydronephrosis is a common anatomical change in the evolution of pregnancy, occurring in $68-100 \%$ of cases of pregnant women after the 20th week of pregnancy (5-7). Sometimes, unilateral or bilateral ureterohydronephrosis (UHN) may be a pathological sign of obstruction due to lithiasis or inflammatory diseases with stenosis, such as tuberculosis. In the present study, 58 cases of pregnant women presenting UHN were included. Depending on the trimester of pregnancy, the frequency was $24.14 \%$ (14 cases) in the second trimester and $74.86 \%$ (44 cases) in the first trimester. No cases of UHN were identified in the first trimester of pregnancy. These data confirmed the literature evidence highlighting that UHN is rare in the first part of the pregnancy, and more frequent in the second and third trimester of pregnancy $(2,5,6,8)$.

Abdominal ultrasonography is the most common imaging examination used during pregnancy, offering important information about kidneys such as anatomical changes, location of UHN, degree of echogenicity of the renal parenchyma and presence of renal and ureteral stones. Other imaging methods can be used, such as MRI, but ultrasound is the most widely used due to the fact there is no irradiation for mother and fetus, also being a relatively 


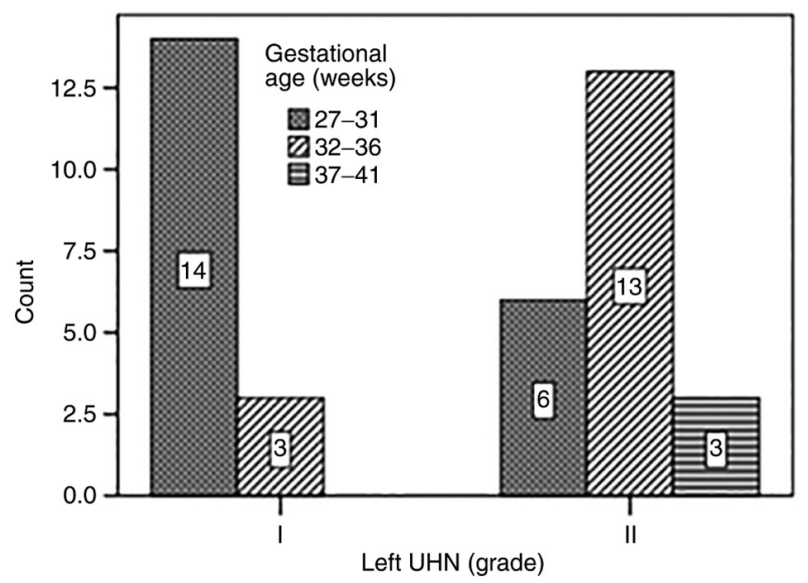

Figure 5. Distribution of left UHN depending on grade and gestational age (weeks). UHN, ureterohydronephrosis.

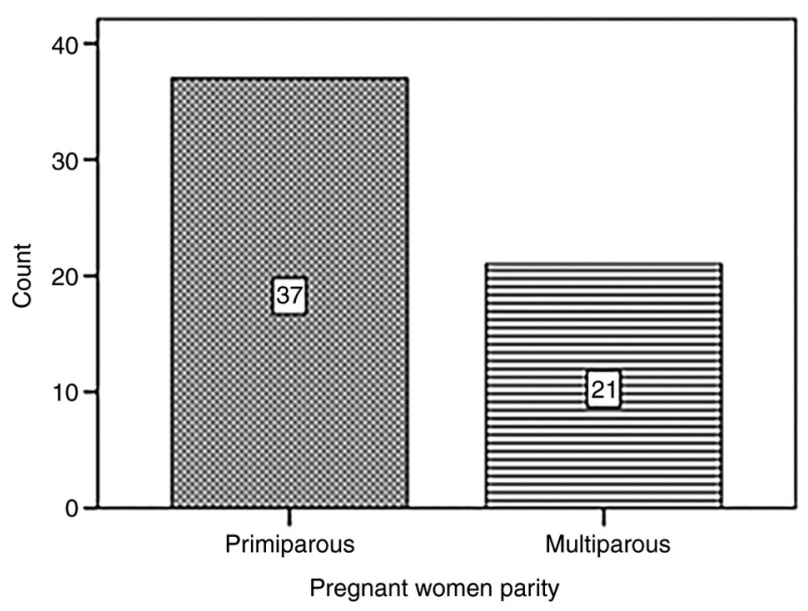

Figure 6. Distribution of studied group depending on parity.

inexpensive technique, with high availability (9). In the present study, ultrasound examination was performed in all 58 pregnant women. Right UHN was present in all pregnant women included in the study, and left UHN was present in $67.24 \%$ (39 cases). These findings confirm the previously reported data according to which UHN has an increased frequency in pregnancy, being more predominant on the right side $(1,10-12)$.

The literature suggests the following physiological factors responsible for UHN occurrence in pregnancy: i) hormonal factors: progesterone relaxes the ureteral smooth muscle (3); ii) obstructive factors: extrinsic ureteral compression, anatomical changes being more obvious on the right side due to the oblique axis of the uterus, but also because of the anatomical position of the ureters in the pelvic area (13); iii) dilated iliac vessels: enlargement of the uterus leading to compression of the iliac vessels resulting in their dilation, and ureteral compression (14); iv) parity: UHN is more pronounced in primiparous women, possibly due to lax tissue muscle, which is more developed in multiparous women (15).

The anatomical changes are more obvious on the right kidney; literature data suggest a frequency of over $85 \%$ of the cases $(10,16)$. In the studied group, it was observed that right UHN was detected in all 58 cases (100\%), compared with the

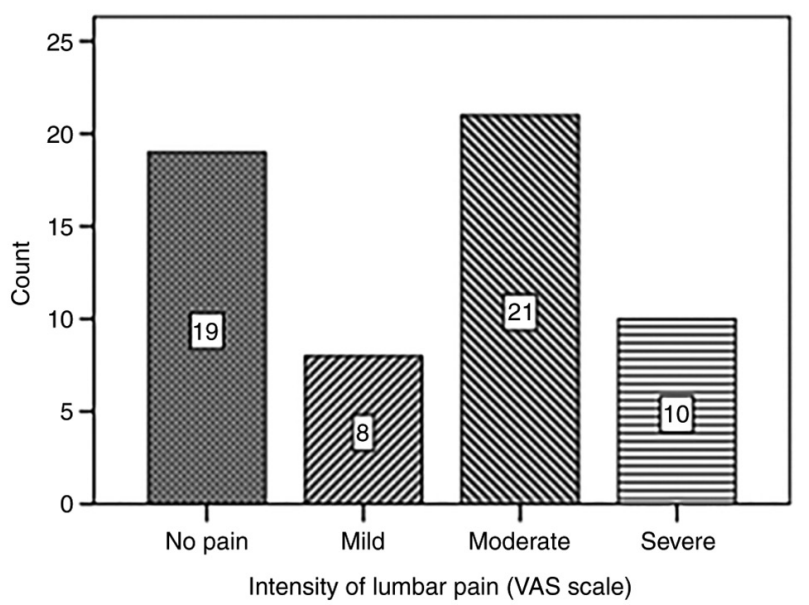

Figure 7. Distribution of the studied group depending on the intensity of lumbar pain (VAS scale).

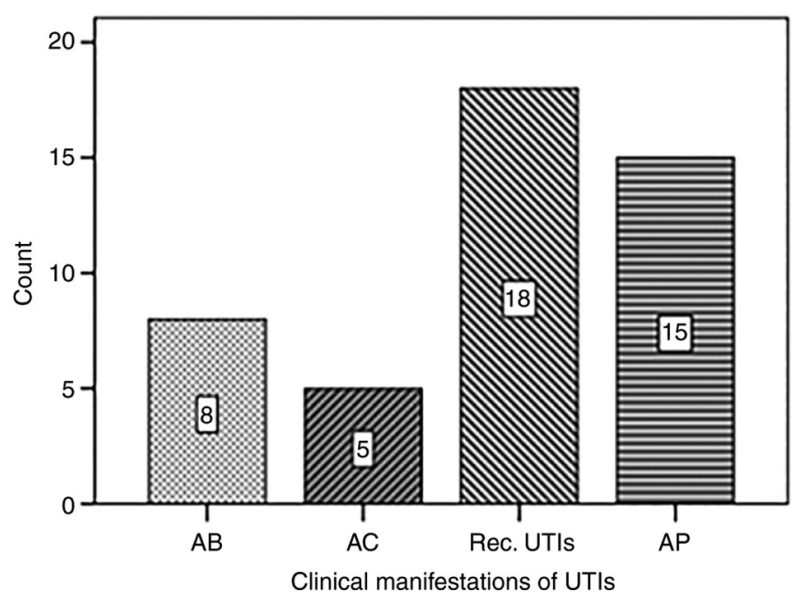

Figure 8. Distribution of clinical manifestations of UTIs in the studied group. UTIs, urinary tract infections; $\mathrm{AB}$, asymptomatic bacteriuria; $\mathrm{AC}$, acute cystitis; Rec. UTIs, recurrent UTIs; AP, acute pyelonephritis.

left UHN (67.24\%), or associated with the right UHN in the studied group. These findings could be due to the dextrorotation of the pregnant uterus and the compression of the right ureter by the iliac and ovarian vessels (17). The right ureter crosses the iliac and ovarian vessels at a narrower angle in the pelvic area than the angle of left ureter, which runs parallel to them (14). Although it is believed that the effect of progesterone in the smooth muscle relaxation may explain the dilation of the pyelocaliceal system, this theory cannot explain the asymmetric dilation of the two uretero-pelvic regions $(17,18)$. Depending on the side and grade of UHN, frequency of grade III was $63.76 \%$ and grade II was $36.21 \%$ in the 58 pregnant women with right UHN; the frequency of grade II was $56.21 \%$ and grade I was $43.59 \%$ in 39 pregnant women with left UHN. It is observed again that the right side is more affected, including grade III of UHN, a grade that is not observed at the left side, with no cases of grade III described.

Our data suggest that grade of left and right UHN increased with gestational age, this fact in the literature data being attributed to the increase of uterine volume, as the pregnancy progresses. Sala and Rubi in their study (17) also 
suggested extrinsic compression as a cause of UHN during pregnancy. Our studied group included UHN grade I, II or III, there were no cases of grade IV, which is in agreement with Karabulut et al (9) and Dawood et al (18).

Findings suggest that UHN appears more frequently in the primiparous pregnant women compared to multiparous pregnant women $(7,19,20)$. Analyzing the data from the present study, the frequency of primiparous was $63.79 \%$ (37 pregnant women) and multiparous was $36.21 \%$ (21 pregnant women) from a total of 58 patients, which suggests that primiparous are more susceptible to develop UHN. The literature does not provide clear explanations for this phenomenon, but according to author Dwan the renal system of primiparous pregnant women has less lax muscle tissue than multiparous women (15). This possible association of UHN with patient's parity was also described in the study of Faundes et al. A significant association was found only in the third trimesters, suggesting that abdominal muscles of primiparous women are more tonic, which leads to compression of the ureters (2).

The most common symptom reported in UHN is lumbar pain on the affected kidney (7). According to the VAS scale, in our studied group $19 \%$ were asymptomatic, $13.79 \%$ had mild pain, $36.21 \%$ moderate pain and $17.24 \%$ had severe lumbar pain. From the 58 pregnant women, $79.31 \%$ (46 cases) were complicated with urinary tract infections (UTI) with different clinical manifestations. The most frequent clinical manifestation was recurrent UTIs ( $31.03 \%$; 18 cases) followed by acute pyelonephritis $(25.86 \% ; 15$ cases). There were also asymptomatic bacteriuria $(13.79 \% ; 8$ cases) and acute cystitis (8.62\%; 5 cases). Acute pyelonephritis and recurrent urinary tract infections are the most frequent clinical manifestations associated with UHN described in the literature during pregnancy $(7,21,22)$. Symptomatic UHN may also be associated with acute renal insufficiency or ureteral stones as complications of this condition $(21,23)$. In the present study, we found $8(13.79 \%)$ pregnant women with ureteral lithiasis and $7(12.07 \%)$ pregnant women with acute kidney insufficiency (AKI), as complications of UHN.

The majority of cases of symptomatic UHN during pregnancy have a positive response on conservative treatment, only $10 \%$ requiring urological surgery with stent insertion due to significant lumbar pain (7). Among the studied group $96.55 \%$ (56 cases) of the studied group, responded to analgesic therapy, antibiotics and hydration, supporting existing data from the literature. Improvement occurs within 2-3 days after starting the treatment $(7,21)$. If no improvement is evident, urological surgery with stent insertion under spinal anesthesia with cystoscopic guidance or Cesarean delivery if the fetal development condition allows $(23,24)$. From the studied group, only 2 pregnant women $(3.45 \%)$ with severe lumbar pain needed stent insertion, which ameliorated the symptomatology that initially did not respond to conservatory treatment. Once the ureteral stent is inserted, it can be left in position for 20 weeks and removed after birth (25). The ureteral stent is usually well tolerated by pregnant women; there are only few cases that require their removal due to complications such recurrent infections or pelvic pain (25). The 2 cases of pregnant women with UHN and stent insertion did not have severe complications, such as urosepsis, the ureteral stents being removed immediately after delivery.
Abdominal ultrasonography has limits in detecting ureteral anatomical changes, especially in obese pregnant women with abdominal meteorism, which can lead to kidney image misinterpretation. CT scan has a $100 \%$ specificity for visualizing kidney stones in all areas of the urinary tract, but in pregnant women cannot be used because of its maternal and fetal risks. MRI does not use ionizing radiation like CT scan and has lower accuracy in detecting small stones (26). The limitation of the present study was the use of ultrasound in all 58 cases, and no MRI investigations in order to have a more clear etiology of urinary tract obstruction in selected cases (uterine compression/kidney stones).

Another limitation of the present study was the non-homogeneous collection of used data registration, because we had to register mixed data, obtained from medical files of hospitalizations of pregnant women, combined with electronical documents from the 'Constanta County Emergency Hospital' and/or other laboratories and private hospitals where patients were checked before. In these situations, some data could be omitted, leading to underestimation of some complications such as urinary tract infection or spontaneous elimination of kidney stones.

In summary, ureterohydronephrosis is a common anatomical change in pregnancy and is dependent on the gestational age, but could be a pathological condition, associated with the ureteral migration of renal stones. The grade of right or left UHN increases progressively with gestational age. Parity influences the development of UHN; primiparous pregnant women are more susceptible to develop a more severe grade of UHN. The most common symptom of hydronephrosis during pregnancy is lumbar pain, which can have different types of intensity (usually moderate to severe). UHN may be complicated with lower or upper urinary tract infection, or even with acute renal insufficiency, a situation that requires urgent, appropriate management. Ureteral stone can be another complication and requires a different approach. Conservatory treatment during symptomatic and complicated UHN is efficient in most cases, otherwise urological interventions with ureteral stent insertion must be initiated, as these treatments are effective and safe both for mother and child.

\section{Acknowledgements}

This work was supported by the project ANTREPRENORDOC, in the framework of Human Resources Development Operational Programme 2014-2020.

\section{Funding}

The study was financed by the European Social Fund under the contract number 36355/23.05.2019 HRD OP/380/6/13-SMIS Code: 123847.

\section{Availability of data and materials}

Data used in the current original study were obtained from patient archive files at Constanta County Emergency Hospital, Romania. Any further information regarding the present study is available from the corresponding author upon reasonable request. 


\section{Authors' contributions}

EC established the design of the study and collected data from patients included in the study. AMPC and LCP contributed to the analysis and interpretation of the data and performed the statistics. AMPC was involved in the drafting of the manuscript. EC and LAT contributed to analysis and writing of the Results and Discussion sections including the literature data and translated it and prepared for publishing. LAT and LCP confirm the authenticity of data. EC, LAT, AMPC and LCP read and approved the final manuscript.

\section{Ethics approval and consent to participate}

The data of the present study were approved by the local Ethics Commission of Constanta County Emergency Hospital, Romania (no. 3/24.02.2017).

\section{Patient consent for publication}

Not applicable.

\section{Competing interests}

The authors declare that they have no competing interests.

\section{References}

1. Fried AM, Woodring JH and Thompson DJ: Hydronephrosis of pregnancy: A prospective sequential study of the course of dilatation. J Ultrasound Med 2: 255-259, 1983.

2. Faundes A, Bricola-Filho M and Pinto e Silva JL: Dilatation of the urinary tract during pregnancy: Proposal of a curve of maximal caliceal diameter by gestational age. Am J Obstet Gynecol 178: 1082-1086, 1998.

3. Schulman A and Herlinger H: Urinary tract dilatation in pregnancy. Br J Radiol 48: 638-645, 1975.

4. Onen A: Grading of hydronephrosis: An ongoing challenge. Front Pediatr 8: 458, 2020.

5. Meares EM Jr: Urologic surgery during pregnancy. Clin Obstet Gynecol 21: 907-920, 1978

6. Fainstat T: Ureteral dilatation in pregnancy: A review. Obstet Gynecol Surv 18: 845-860, 1963.

7. Puškar D, Balagović I, Filipović A, Knezović N, Kopjar M, Huis $\mathrm{M}$ and Gilja I: Symptomatic physiologic hydronephrosis in pregnancy: Incidence, complications and treatment. Eur Urol 39: 260-263, 2001

8. The $\mathrm{S}$ and Chan L: OP21.05: Pelvicalyceal dilatation in maternal kidneys during normal pregnancy-an uncommon finding? Ultrasound Obstet Gynecol 30: 529-529, 2007.
9. Karabulut N, Baki Yağci A and Karabulut A: Renal vein Doppler ultrasound of maternal kidneys in normal second and third trimester pregnancy. Br J Radiol 76: 444-447, 2003.

10. Peake SL, Roxburgh HB and Langlois SL: Ultrasonic assessment of hydronephrosis of pregnancy. Radiology 146: 167-170, 1983.

11. Cietak KA and Newton JR: Serial qualitative maternal nephrosonography in pregnancy. Br J Radiol 58: 399-404, 1985.

12. Ciciu E, Pasatu-Cornea AM and Tuta LA: Mo117 the impact of gestational age on anatomical and physiological changes of the upper urinary tract during pregnancy. Nephrol Dialysis Transplantation: May 29, 2021 (Epub ahead of print).

13. Rasmussen PE and Nielsen FR: Hydronephrosis during pregnancy: A literature survey. Eur J Obstet Gynecol Reprod Biol 27: 249-259, 1988

14. Cheung KL and Lafayette RA: Renal physiology of pregnancy. Adv Chronic Kidney Dis 20: 209-214, 2013.

15. Dawn CS: Textbook of Obestetrics and Neonatology. 11th edition. Pratap Medical Publishers, New Delhi, 1995.

16. Schneider DH, Eichner E and Gordon MB: An attempt at production of hydronephrosis of pregnancy, artificially induced. Am J Obstet Gynecol 65: 660-665, 1953.

17. Sala NL and Rubí RA: Ureteral function in pregnant women. II. Ureteral contractility during normal pregnancy. Am J Obstet Gynecol 99: 228-236, 1967

18. Dawood S, Amin S and ShekhMuhammed S: Sonographic evaluation of maternal kidneys in normal pregnancy. Zanco J Med Sci 19: 880-885, 2015.

19. Eckford SD and Gingell JC: Ureteric obstruction in pregnancy-diagnosis and management. Br J Obstet Gynaecol 98: 1137-1140, 1991.

20. Zwergel T, Lindenmeir T and Wullich B: Management of acute hydronephrosis in pregnancy by ureteral stenting. Eur Urol 29: 292-297, 1996.

21. Tsai YL, Seow KM, Yieh CH, Chong KM, Hwang JL, Lin YH and Huang LW: Comparative study of conservative and surgical management for symptomatic moderate and severe hydronephrosis in pregnancy: A prospective randomized study. Acta Obstet Gynecol Scand 86: 1047-1050, 2007.

22. McGready R, Wuthiekanun V, Ashley EA, Tan SO, Pimanpaanrak M, Viladpai-Nguen SJ, Jesadapanpong W, Blacksell SD, Proux S, Day NP, et al: Diagnostic and treatment difficulties of pyelonephritis in pregnancy in resource-limited settings. Am J Trop Med Hyg 83: 1322-1329, 2010.

23. Rosenberg E, Sergienko R, Abu-Ghanem S, Wiznitzer A, Romanowsky I, Neulander EZ and Sheiner E: Nephrolithiasis during pregnancy: Characteristics, complications, and pregnancy outcome. World J Urol 29: 743-747, 2011.

24. Ceçen K and Ülker K: The comparison of double J stent insertion and conservative treatment alone in severe pure gestational hydronephrosis: A case controlled clinical study. ScientificWorldJournal 2014: 989173, 2014.

25. Haleblian G, Kijvikai K, de La Rosette J and Preminger G: Ureteral stenting and urinary stone management: A systematic review. J Urol 179: 424-430, 2008.

26. MacNeily AE, Goldenberg SL, Allen GJ, Ajzen SA and Cooperberg PL: Sonographic visualization of the ureter in pregnancy. J Urol 146: 298-301, 1991. 